\title{
Outcomes Following Negative Prostate Biopsy for Patients with Persistent Disease after Radiotherapy for Prostate Cancer
}

\author{
Jacob H. Cohen, James Eastham, Richard J. Macchia
}

Department of Urology (JHC, RJM), State University of New York Downstate Medical School, Brooklyn, New York, USA, and Memorial Sloan-Kettering Cancer Center (JE), New York, New York, USA

\begin{abstract}
Purpose: When faced with biochemical recurrence after definitive radiotherapy for prostate cancer, clinicians must determine whether the recurrence is local or systemic. Post radiotherapy prostate biopsies to detect persistent local disease are difficult to interpret histopathologically and are subject to sampling error. Our study examines outcomes for patients with a negative prostate biopsy performed for rising prostate-specific antigen (PSA) levels after prostate radiation.

Materials and Methods: We performed a retrospective review of 238 prostate cancer patients with a negative biopsy following definitive radiotherapy. Seventy-five of these patients had biochemical recurrence at the time of biopsy. A negative biopsy was defined as the absence of prostate cancer without radiation-treatment effect in the specimen.

Results: Patients underwent biopsy at a mean of 41 months after the completion of radiation. They had a mean PSA of 6. Patients were followed for an average of 63 months. Thirty-two patients $(43 \%)$ developed metastasis, and $11(15 \%)$ died of prostate cancer despite a negative post-radiation biopsy. Five of nine patients (56\%) with sequential biopsies had a positive second biopsy.

Conclusions: Patients with PSA recurrence and a negative post-radiation biopsy have a high chance of persistent local disease, progression, and death from prostate cancer. Furthermore, an initial negative biopsy does not rule-out local recurrence. Patients with biochemical recurrence after radiotherapy for prostate cancer need to be evaluated earlier for local recurrence.
\end{abstract}

Key words: prostate neoplasms; prostate-specific antigen; neoplasm recurrence; radiation

Int Braz J Urol. 2010; 36: 44-8

\section{INTRODUCTION}

Biochemical recurrence after radiotherapy for prostate cancer occurs in approximately $10-60 \%$ of patients and varies depending on definition of recurrence, tumor stage and grade at the time of diagnosis, dosage of radiation, and the use of adjuvant hormonal therapy (1). Salvage therapy for persistent local disease after radiotherapy has shown greatest efficacy for biopsy-proven local recurrence with low prostate-specific antigen (PSA) level, and negative metastatic evaluation (2). Post-radiotherapy prostate biopsy to detect persistent local disease is difficult to interpret histopathologically and subject to sampling error. In addition, there are no well-defined recommendations for when or how to biopsy these patients. Most studies have examined post-radiotherapy biopsies regardless of signs or symptoms or disease progression. Our study is the first, to our knowledge, to examine outcomes for patients with a negative 
prostate biopsy performed for biochemical recurrence after radiation.

\section{MATERIALS AND METHODS}

We performed a retrospective review of 238 prostate cancer patients in a prospectively maintained prostate cancer database who had a negative prostate biopsy following definitive radiotherapy between January 1st, 1992 and December 31st, 2005. Of these patients, 155 underwent prostate biopsy as part of a clinical trial without evidence of biochemical failure, while 83 patients were identified to have biochemical recurrence at the time of their post-radiotherapy biopsy. Eight patients were excluded due to missing data or lack of follow-up, leaving 75 patients for analysis. Biochemical recurrence was determined by the treating practitioner, most typically the American Society for Therapeutic Radiology and Oncology (ASTRO) definition of biochemical failure (3). A negative postradiation biopsy included a pathology report of benign tissue, benign tissue with profound treatment effect, or prostate cancer with profound treatment effect (4).

\section{RESULTS}

Our 75 patients had a mean age of 66 years and a mean PSA of $15 \mathrm{ng} / \mathrm{mL}$ at initial cancer diagnosis. Table-1 shows Gleason score and clinical stage at initial cancer diagnosis as well as initial treatment. A post-radiotherapy PSA-nadir of $<1.0$ was achieved in 69 patients $(92 \%)$. Patients underwent biopsy at a mean of 41 months after the completion of radiation and with a mean PSA of $6 \mathrm{ng} / \mathrm{mL}$. Mean and median PSA doubling time at post-radiotherapy biopsy were 12 and 9 months, respectively. For the 55 (73\%) patients on whom data were available, there was no standard technique for post-radiotherapy biopsy, with as little as 2 cores and as many as 24 cores sampled. Mean follow-up after the negative post-radiotherapy biopsy was 63 months.

Patient outcomes following their negative post-radiation biopsy are presented in Figure-1. There were nine patients undergoing two sequential postradiotherapy biopsies. Five of nine $(56 \%)$ had their second biopsy return positive, and all five were alive after their salvage local therapies. Of the four patients who had two sequential negative post-radiotherapy biopsies, there was one death from prostate cancer, one patient with clinical metastasis, one patient with asymptomatic metastasis, and one patient with biochemical recurrence alone.

Overall, 32 patients (43\%) developed disease progression beyond biochemical recurrence, with 15 $(20 \%)$ developing radiographic metastasis only, 6 (8\%) developing clinically symptomatic metastasis, and $11(15 \%)$ dying from prostate cancer. These patients had a mean PSA of 9 at the time of re-biopsy, with a mean PSA-doubling time of 9 months.

Twenty-nine patients (39\%) had biochemical recurrence only and were observed. Their mean PSA

Table 1 - Clinical and treatment parameters.

\begin{tabular}{lcc}
\hline & N & \% \\
\hline Gleason score & & \\
$\begin{array}{l}3+2=5 \\
3+3=6\end{array}$ & 4 & 5 \\
$3+4=7$ & 26 & 35 \\
$4+3=7$ & 21 & 28 \\
$4+4=8$ & 12 & 16 \\
$5+4=9$ & 9 & 12 \\
Clinical stage & 3 & 4 \\
T1c & & \\
T2 & 35 & 47 \\
T3 & 30 & 40 \\
T4 & 9 & 12 \\
Radiation dose & 1 & 1 \\
EBRT to 8100 & & \\
EBRT to 7700 & 13 & 17 \\
EBRT to 7560 & 3 & 4 \\
EBRT to 7020 & 20 & 27 \\
EBRT to 6600 & 15 & 20 \\
EBRT/brachytherapy & 4 & 5 \\
Unknown dose & 15 & 20 \\
Hormones used & 7 & 9 \\
Hormones not used or missing data & 36 & 48 \\
\hline
\end{tabular}

$E B R T=$ external beam radiation. 


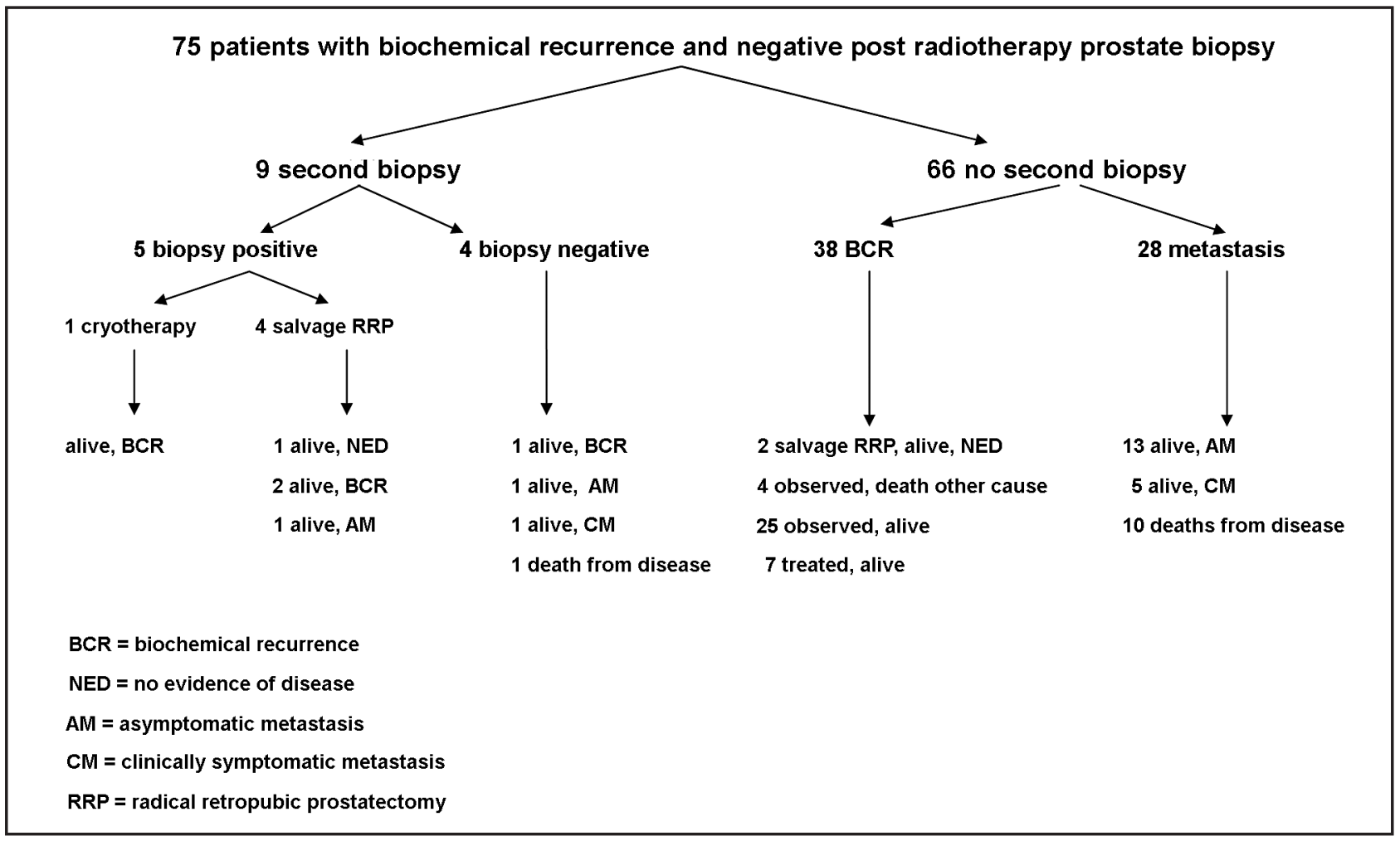

Figure 1-Clinical outcomes.

at the time of re-biopsy was 4 , with a mean PSAdoubling time of 15 months. This group of patients was followed for a mean 56 months after re-biopsy, and the mean PSA at last follow-up was 11 with four deaths from other causes.

\section{COMMENTS}

Post-radiotherapy biopsies are very complex to interpret and depend on the experience of the reading pathologist, the elapsed time interval following radiotherapy, the presence or absence of concomitant androgen therapy, the total dose of radiotherapy administered, and the amount and degree of treatment effect. Radiotherapy is known to induce a variety of histological changes in normal and cancerous prostate tissue, including atrophy, cytology atypia, mucinous metaplasia, cytoplasmic vacuolization, and diminution of neoplastic glands (4).

Complicating matters, the effect of radiation on prostate cancer cells changes over time. Crook et al. prospectively studied 498 patients who underwent systematic 6-core trans-rectal ultrasound-guided prostate biopsies at standard intervals following radiotherapy (at 12 months post radiation and every 6-12 months thereafter) (5). Thirty percent of patients with an initially positive first post-treatment biopsy at 12 months eventually converted to a negative biopsy at a mean time of 30 months. Indeterminate biopsies (those with profound treatment effect) at first posttreatment biopsy converted to negative in $30 \%$ and progressed to local failure in $18 \%$. Finally, $19 \%$ of those with an initially negative post-treatment biopsy were found to have residual local disease at systematic 36-month biopsy. The authors concluded that the greatest predictive value of a post-radiation biopsy is between 30 and 36 months, and that biopsies with profound treatment effect (intermediate category) should be repeated as residual radiated tumor eventually declares its biological activity over time.

Positive re-biopsy rates have also been shown to correlate with radiation dose. Liebel et al. found lower positive re-biopsy rates at 2.5 years after ra- 
diation for higher radiation doses, with $57 \%$ positive re-biopsy rate at $64.8 \mathrm{~Gy}, 44 \%$ at $70.2 \mathrm{~Gy}, 45 \%$ at $75.6 \mathrm{~Gy}$, and $7 \%$ at $81 \mathrm{~Gy}(6)$. Zelefsky et al. also corroborated these findings (7). In contrast, Pollack et al. found equal positive re-biopsy rates when comparing 70-Gy to 78-Gy (8).

In 1999, ASTRO published a consensus statement regarding guidelines for re-biopsy after radiation (9). This group concluded that systematic prostate re-biopsy is not a standard of care for prostate cancer patients, that it should only be considered for patients who are candidates for effective salvage local therapy, and that it should be performed at least two years following the conclusion of radiation therapy. PSA guidelines were not given in this statement. A later consensus statement showed the newer Phoenix definition of recurrence (nadir plus 2) to predict for metastatic failure, implying that biopsies to detect persistent local disease should occur before "nadir plus 2" is reached (10).

Many studies have shown increased incidence of local recurrence, distant metastasis, and death from prostate cancer for those patients with a positive post-radiation biopsy $(11,12)$. Our retrospective study specifically examined outcomes for patients with a negative post-radiation prostate biopsy. Decision to re-biopsy was at the discretion of the treating physician, but all patients had a rising PSA at the time of re-biopsy.

The patients in our study had generally poor outcomes, with 32 (43\%) developing clinical disease progression. All of these patients were treated with hormonal therapy, and an additional 14 patients received hormonal therapy based on biochemical recurrence alone. Indeed, our patients had high PSA levels at the time of re-biopsy (mean 6) and high PSA doubling times at the time of re-biopsy (mean 12 months), indicating that the initiation of the search for persistent local disease occurred relatively late in the disease process. Not surprisingly, those patients with the worst outcomes (radiographic or clinical metastasis or death from prostate cancer), had higher mean PSA (9) and shorter mean PSA-doubling time ( 9 months) at the time of their biopsy. Another possible contributing factor to the diverse clinical outcomes we observed might be the wide range of radiotherapy doses used during initial treatment, as well as whether neoadjuvant or adjuvant hormonal therapy was used.

Our retrospective review does not allow definitive statements regarding the prognostic value of a negative post-radiation biopsy performed for biochemical recurrence. However, it is interesting that despite five of nine patients $(56 \%)$ of those undergoing multiple biopsies being upgraded to cancer on repeat biopsy, all of these patients were successfully salvaged with local therapy, and two of these patients had nodal metastasis at the time of salvage prostatectomy. Had these patients not been re-biopsied, they may not have been offered successful local/regional salvage therapy, and their disease outcome would have likely suffered.

In conclusion, we documented high rates of disease progression and eventual death from prostate cancer in a group of 75 men who had rising PSA after radiotherapy but a negative post-radiation prostate biopsy. In future studies, we will compare outcomes to patients in the same database with positive postradiation prostate biopsies. In clinical practice, we believe men with rising PSA after radiation should be offered a systematic prostate biopsy to document persistent local disease and offer the possibility of cure with additional local/regional therapy.

\section{CONFLICT OF INTEREST}

None declared.

\section{REFERENCES}

1. Khuntia D, Reddy CA, Mahadevan A, Klein EA, Kupelian PA: Recurrence-free survival rates after external-beam radiotherapy for patients with clinical T1-T3 prostate carcinoma in the prostate-specific antigen era: what should we expect? Cancer. 2004; 100 : 1283-92.

2. Link P, Freiha FS: Radical prostatectomy after definitive radiation therapy for prostate cancer. Urology. 1991; 37: 189-92.

3. No authors listed: Consensus statement: guidelines for PSA following radiation therapy. American Society for Therapeutic Radiology and Oncology Consensus Panel. Int J Radiat Oncol Biol Phys. 1997; 37: 1035-41. 
4. Gaudin PB, Zelefsky MJ, Leibel SA, Fuks Z, Reuter VE: Histopathologic effects of three-dimensional conformal external beam radiation therapy on benign and malignant prostate tissues. Am J Surg Pathol. 1999; 23: 1021-31.

5. Crook J, Malone S, Perry G, Bahadur Y, Robertson S, Abdolell M: Postradiotherapy prostate biopsies: what do they really mean? Results for 498 patients. Int $\mathrm{J}$ Radiat Oncol Biol Phys. 2000; 48: 355-67.

6. Leibel SA, Zelefsky MJ, Kutcher GJ, Burman CM, Mohan R, Mageras GS, et al.: The biological basis and clinical application of three-dimensional conformal external beam radiation therapy in carcinoma of the prostate. Semin Oncol. 1994; 21: 580-97.

7. Zelefsky MJ, Fuks Z, Hunt M, Lee HJ, Lombardi D, Ling CC, et al.: High dose radiation delivered by intensity modulated conformal radiotherapy improves the outcome of localized prostate cancer. J Urol. 2001; 166: 876-81. Erratum in: J Urol. 2001; 166: 1839.

8. Pollack A, Zagars GK, Antolak JA, Kuban DA, Rosen II: Prostate biopsy status and PSA nadir level as early surrogates for treatment failure: analysis of a prostate cancer randomized radiation dose escalation trial. Int J Radiat Oncol Biol Phys. 2002; 54: 677-85.
9. Cox JD, Gallagher MJ, Hammond EH, Kaplan RS, Schellhammer PF: Consensus statements on radiation therapy of prostate cancer: guidelines for prostate rebiopsy after radiation and for radiation therapy with rising prostate-specific antigen levels after radical prostatectomy. American Society for Therapeutic Radiology and Oncology Consensus Panel. J Clin Oncol. 1999; 17: 1155.

10. Roach M 3rd, Hanks G, Thames H Jr, Schellhammer P, Shipley WU, Sokol GH, et al.: Defining biochemical failure following radiotherapy with or without hormonal therapy in men with clinically localized prostate cancer: recommendations of the RTOG-ASTRO Phoenix Consensus Conference. Int J Radiat Oncol Biol Phys. 2006; 65: 965-74.

11. Kuban DA, el-Mahdi AM, Schellhammer P: The significance of post-irradiation prostate biopsy with long-term follow-up. Int J Radiat Oncol Biol Phys. 1992; 24: 409-14.

12. Zelefsky MJ, Reuter VE, Fuks Z, Scardino P, Shippy A: Influence of local tumor control on distant metastases and cancer related mortality after external beam radiotherapy for prostate cancer. J Urol. 2008; 179: 1368-73; discussion 1373.

\author{
Correspondence address: \\ Dr. Jacob H. Cohen \\ Division of Urology \\ State University of New York \\ Downstate Medical School \\ Brooklyn, NY, 11203, USA \\ Fax: + 1718 270-3848 \\ E-mail: jhcohen4@gmail.com
}

